\title{
Dynamic response of an accelerator driven system to accelerator beam interruptions for criticality
}

\author{
A. Lafuente, A. Abanades, P.T. Leon, F. Sordo, J.M. Martinez-Val \\ E.T.S. Ingenieros Industriales, J. Gutierrez Abascal, 2-28006 Madrid, Spain
}

\begin{abstract}
Subcritical nuclear reactors driven by intense neutron sources can be very suitable tools for nuclear waste transmutation, particularly in the case of minor actinides with very low fractions of delayed neutrons. A proper control of these systems needs to know at every time the absolute value of the reactor subcriticality (negative reactivity), which must be measured by fully reliable methods, usually conveying a short interruption of the accelerator beam in order to assess the neutron flux reduction. Those interruptions should be very short in time, for not disturbing too much the thermal magnitudes of the reactor. Otherwise, the cladding and the fuel would suffer from thermal fatigue produced by those perturbations, and the mechanical integrity of the reactor would be jeopardized. It is shown in this paper that beam interruptions of the order of $400 \mu$ s repeated every second would not disturb significantly the reactor thermal features, while enabling for an adequate measurement of the negative reactivity.
\end{abstract}

Keywords: Accelerators; Subcritical systems; Beam interruptions; Reactivity

\section{Introduction and background}

One of the most challenging scientific subjects in nuclear energy research is the quest for the reduction of its longterm environmental impact by minimizing the radiotoxicity of long-lived radioactive waste inventory. Such objective is sought by inducing nuclear transmutation in the relevant radioactive nuclei of the nuclear waste. Accelerator driven systems (ADS) are one of the most promising concepts under development for this purpose

Such systems are based in the operation of a subcritical nuclear reactor, in which the neutrons needed to maintain the neutron flux, and therefore the thermal power, are provided by a high power external neutron source A spallation target is the preferred device as external neutron source, usually consisting in a heavy material (as lead or lead-bismuth eutectic (LBE)) in which medium energy protons (some hundred $\mathrm{MeV}$ ) impinge and produce neutrons by spallation phenomena Particle accelerators are used to accelerate proton beams up to the energy required to activate the intense neutron source. Those neutrons drive the subcritical core up to the required flux level, and the corresponding level of neutron-induced transmutation reactions.

An ADS thus involves three main components: the accelerator, the spallation target and the subcritical reactor. Those components can be developed separately, but they must be coupled for the full operation of an ADS, and they must be analyzed as a chain in order to characterize the ADS behavior and to asses its internal coherence. Several works have been carried out to describe the behavior of a subcritical device, including safety analysis to account for reactivity insertion accidents, kinematic response of the system, and so forth

In particular, the target must be studied from the point of view of its physical integrity. It will be operating at high temperatures and under strong thermal stresses. It will also 
suffer from radiation damage and corrosion, and several nuclear reactions will take place inside it, so appearing new nuclei, particularly hydrogen and helium. Of course operational safety studies will be needed to assess the compliance of the target with nuclear safety and radiation protection standards in a given ADS

The accelerator is a complex machine that should work with a very high reliability to ensure continuous operation of the ADS. Reliability analysis of accelerators for ADS are based on the determination of the total number of failures, or the mean time between failures (MTBF), by a systematic failure component assessment that takes also into account the failure time span

The integration of the accelerator into the ADS system is one of the main aspects of the ADS development, as the accelerator is a key device to control safety and operation of the ADS, through the neutron intensity produced in the target. As the nuclear mechanisms involved in spallation processes are linearly dependent on the number of incoming particles (if the particle energy is kept constant), the source intensity is proportional to the accelerator power. In turn, the thermal power of the ADS depends on the neutron source intensity through the following equation:

$P=c\left\langle\frac{S \Phi^{+}}{-\rho}\right\rangle$

$S$ being the source strength $\left(\mathrm{n} \mathrm{s}^{-1}\right), \rho$ the reactivity defined in terms of the effective neutron multiplication factor of the subcritical reactor, $K_{\text {eff, }}$, by

$\rho=\left(K_{\text {eff }}-1\right) / K_{\text {eff }}$

$\Phi^{+}$is the importance of the source neutrons, and $c$ is a proportionality coefficient that can be determined, both experimentally or theoretically, in a given reactor. Although $\Phi^{+}$can vary because of spectral modifications, most of the power variations will come from fluctuations in the source intensity $S$ and the reactivity value $\rho$.

Monitoring the power level of the plant can rely on a traditional system with fission chambers and thermocouples. However, the innovative part of the control of an ADS comes from actions which could be accomplished via the beam power (source intensity control). For safety reasons, it is very important to perform independent measurements of the source intensity $S$ and the reactivity value $\rho$, in order to confirm the nuclear core subcriticality.

Limitations of the so-called static methods to determine an absolutely calibrated measurement of the criticality level motivated the development of a family of techniques called dynamic methods. In the 1960 s, dynamic methods based on the utilization of time-dependent external neutron sources were proposed These methods have been developed and applied to investigate the physical parameters of neutron-multiplicative systems and to measure the reactivity and kinetic parameters of these systems.
Several dynamic methods (Noise, Source Jerk (SJ), Prompt Jump (PJ), Prompt Neutron Decay, Harmonic Source) have been considered in the recent european PDSXADS project as suitable ones for subcriticality level monitoring Either the classical SJ or the Slope Fit Method methods seem to be the most promising ones to be used in coincidence with periodical short interruptions of the accelerator beam, in order to measure absolutely calibrated values of reactivity as frequently as needed.

Spontaneous complete interruptions of the beam will be detected by the monitoring system of the accelerator proton beam intensity. In coincidence, a dynamic method would be applied from the recordings of the evolution of the neutron flux, and a reactivity measurement would be obtained. In the absence of spontaneous beam interruptions for a long time, a forced short interruption can easily be induced in the accelerator to allow for a quasicontinuous absolute calibration of the subcriticality.

It is worth noting that subcritical devices devoted to transmutation have safety advantages respect to critical reactors with the same type of fuel, because of the subcriticality margin. This is mainly related to the small value of the delayed-neutron fraction of most of the minor actinides ( $\mathrm{Np}, \mathrm{Am}$, and $\mathrm{Cm}$ isotopes). Those nuclei and their descendants are the most offending nuclei as far as long-term radio-toxicity is concerned. The delayed-neutron fraction is 0.0065 for ${ }^{235} \mathrm{U}$ and 0.0023 for ${ }^{239} \mathrm{Pu}$. For ${ }^{241} \mathrm{Am}$ is only 0.0014 and it barely is 0.001 for ${ }^{243} \mathrm{Cm}$. A low value of this fraction means a short reactivity span between delayed-critical and prompt-critical conditions, the latter marking a forbidden level that can lead to a catastrophic accident. A prompt-critical accident happened in Chenobyl-4 in 1986, which is the biggest disaster in the history of the civilian nuclear industry An ADS would be much safer in this context, because it will be designed to remain subcritical always. Obviously, such an important feature will have to be measured when required, to guarantee the reactor subcriticality.

In a subcritical ADS the total interruption of the beam will reduce the reactor thermal power to the level of the decay heat in a very short time, of the order of several prompt neutron lifetimes, i.e., less than $100 \mu$ s in general. The specific value in a given ADS will mainly depend on the fuel composition.

It is worth noting that the speed of the power decline during the beam interruption will be a function of the subcriticality level. This feature enables us to make a calibrated measurement, which would convey a sudden reduction in thermal power. On the contrary, switching-on the beam again up to its nominal value will induce a heating transient.

The aim of this paper is to analyze the impact of these thermal variations produced by the reactivity monitoring techniques in the most sensitive material for the integrity of the ADS, notably the fuel cladding. It is well known that a fundamental criterion of nuclear safety is to keep the 
integrity of the radioactive barriers in any conceivable condition. Therefore, thermal variations produced by beam interruptions must be analyzed to evaluate the mechanical damage they could induce, so that the cladding integrity could be guaranteed by setting limits to the beam interruption process, if needed.

The dynamic response of the ADS as a result of beam interruptions for criticality measurement has been featured as follows:

(a) Calculation of the source neutrons produced by the interaction of a proton beam into the molten metal target. These neutrons must be defined in number and spectrum.

(b) Numerical Monte Carlo calculation of the neutron subcritical multiplication in the reactor, as a response to the beam power variations. This numerical simulation gives the neutron flux level variations, and therefore the thermal power variations in the reactor (and in any fuel pin under consideration).

(c) Thermal-hydraulic analysis of a fuel pin featuring the reactor lattice.

(d) Analysis of thermo-mechanical stresses in the structural material (fuel cladding) as a result of beam interruptions.

In Section 2, the general problem is characterized, particularly the neutron source performance and the subcritical reactor features. A simplified reactor configuration will be used in order to obtain general conclusions on this problem. Of course, for a given reactor or facility, detailed calculations could be done. The main calculation tool would be a Monte Carlo code using reliable neutron reactions data. The numerical results will be interpreted with a simple diffusion theory analytical calculation, which is not as accurate as the numerical simulation, but it is based on an easy-to-follow theory with a clear physical interpretation. In Section 3, the thermal response to beam interruptions will be evaluated. It will include the consideration of fatigue criteria to determine the anticipated operational life of the fuel cladding. In Section 4, some conclusions will be presented, including some guidance for establishing limits to the beam interruption duration, in order to make it possible a quasi-continuous measurement of the subcriticality margin.

\section{Characterization of the problem}

Some key safety issues related to the structural integrity of Accelerator Driven System during normal or abnormal operation are linked to the instabilities, interruptions, or any other perturbations of the proton beam. Every analysis of this problem requires a correct characterization of the neutron source and a suitable model of the reactor core. In the safety analysis of a given design, very accurate and detailed descriptions should be formulated, and very accurate neutronic calculations should be carried out. For a general analysis of the problem, a simplified reactor geometry can be used, with a proper characterization of the reactor composition.

For our study, the reactor has been simplified as a twozone slab depicted in Fig. 1. Take into account that upper and lower boundaries are periodic surfaces, in order to simulate an infinite length in this direction. The third space direction is also infinity (normal to the picture plane).

The inner zone is a mixture of coolant (molten LBE) and fuel, whose isotopic composition has been taken from the PDS-XADS project [20], and it is given in Table 1. It corresponds to a prototype for an actinide burner, still based on uranium oxide matrix. The outer part is a neutron reflector, made just of coolant, that has been introduced in the Monte Carlo simulation in order to fix the subcriticity level to 0.97 . The simplified source in this model is placed in the reactor mid-plane, and it emits the spallation neutrons, defined by their amount and spectrum that we will previously calculate as will be explained in next sections. This simplification opens the possibility of making a comparative study between the results obtained by numerical simulation with MCNPX in the fuel region and the analytical solution of the neutron diffusion equation for a slab as the one depicted in Fig. 1, which helps understand the physics of the problem.

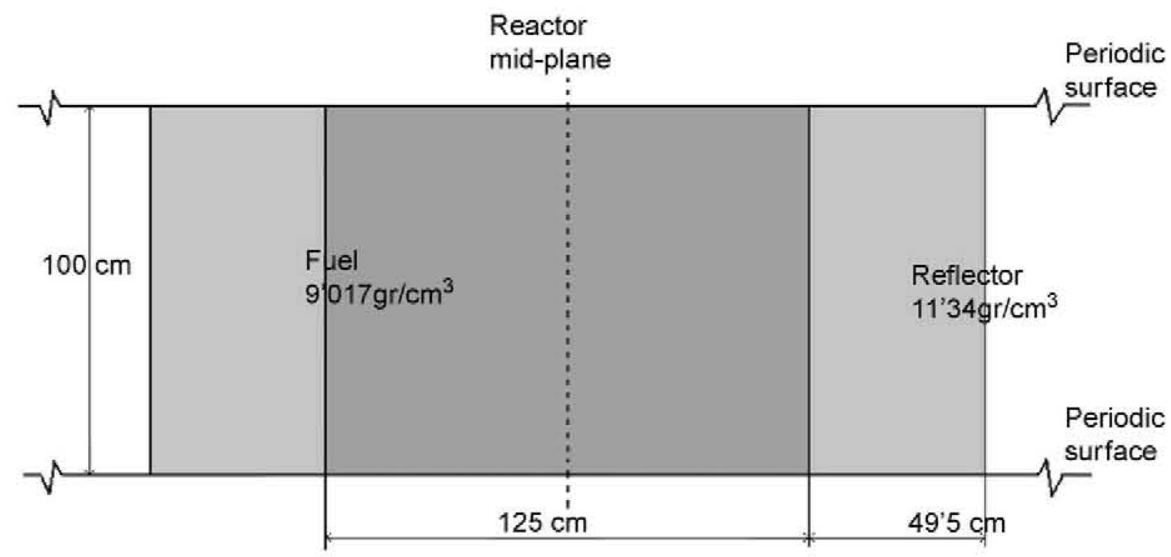

Fig. 1. Slab reactor geometry and dimensions. 
Table 1

Fuel composition

\begin{tabular}{ll}
\hline Isotope & \% weight \\
\hline${ }^{234} \mathrm{U}$ & $9.0214 \mathrm{E}-04$ \\
${ }^{235} \mathrm{U}$ & $2.3805 \mathrm{E}-02$ \\
${ }^{236} \mathrm{U}$ & $1.3417 \mathrm{E}-03$ \\
${ }^{238} \mathrm{U}$ & $4.4092 \mathrm{E}+00$ \\
${ }^{238} \mathrm{Pu}$ & $8.9909 \mathrm{E}-03$ \\
${ }^{239} \mathrm{Pu}$ & $7.8837 \mathrm{E}-01$ \\
${ }^{240} \mathrm{Pu}$ & $2.8377 \mathrm{E}-01$ \\
${ }^{241} \mathrm{Pu}$ & $6.8546 \mathrm{E}-02$ \\
${ }^{242} \mathrm{Pu}$ & $4.1972 \mathrm{E}-02$ \\
${ }^{241} \mathrm{Am}$ & $3.9220 \mathrm{E}-02$ \\
${ }^{16} \mathrm{O}$ & $1.1333 \mathrm{E}+01$ \\
${ }^{206} \mathrm{~Pb}$ & $9.1707 \mathrm{E}+00$ \\
${ }^{207} \mathrm{~Pb}$ & $8.4096 \mathrm{E}+00$ \\
${ }^{208} \mathrm{~Pb}$ & $1.9939 \mathrm{E}+01$ \\
${ }^{209} \mathrm{Bi}$ & $4.5481 \mathrm{E}+01$ \\
\hline
\end{tabular}

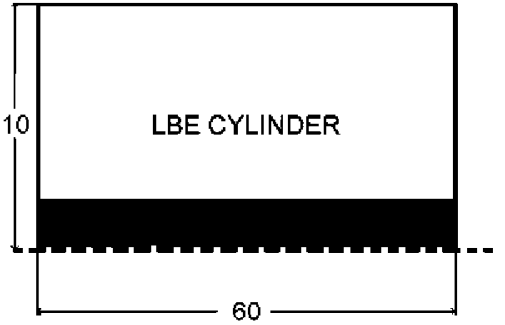

Fig. 2. The spallation source is a LBE cylinder, $60 \mathrm{~cm}$ long and $10 \mathrm{~cm}$ radius. Beam radial intensity $\left(\mu \mathrm{A} / \mathrm{cm}^{2}\right)$ profile is shown in the right-hand side.

Although a simplified source has been used in the one-dimensional reactor model, its features have been calculated using the description of spallation sources usually designed for ADS, as those of the PDS-XADS project. Main features of the source are described below.

\subsection{The neutron source}

The source has been modeled as a cylinder made of LBE (see Fig. 2) irradiated by a beam of $600 \mathrm{MeV}$ protons impinging along the axis with a cross-section distribution as the one given also in Fig. 2. This source has been used to determine the neutron generation rate and spectrum, as well as the angular distribution throughout the different surfaces. These parameters, obtained with MCNPX, have been used to define the simplified source that feeds the core. LBE was chosen as the target material for its remarkable properties as a suitable molten metal coolant with a low neutron capture. On the contrary, it has a high rate of ${ }^{210} \mathrm{Po}$ generation, which is a non-negligible problem of radiological protection (requiring coolant purification and radioactive products confinement).

For the design of the beam we have assumed a current intensity of $6 \mathrm{~mA}$, elliptically distributed, with a spot

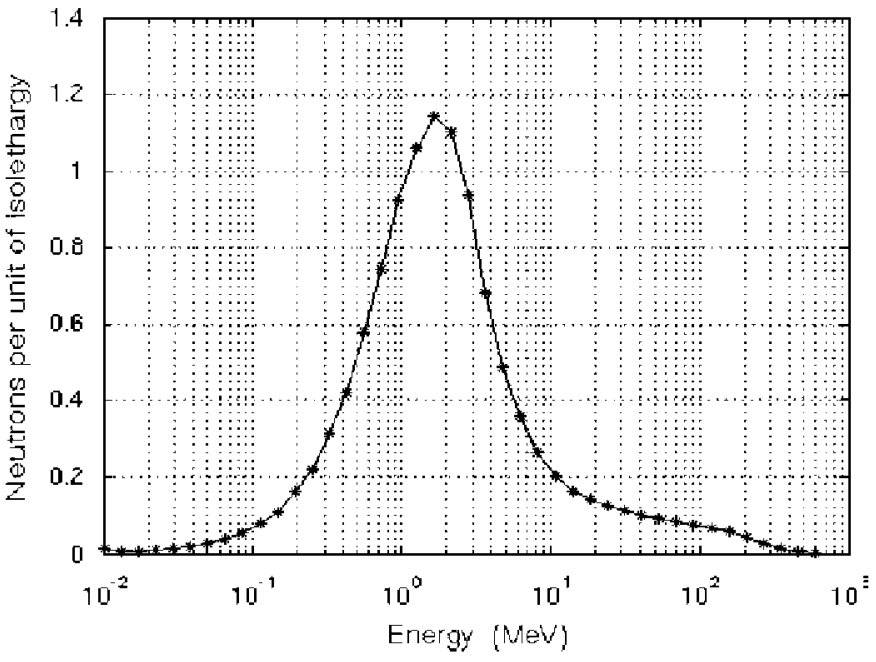

Fig. 3. Neutron spectrum of the spallation target.

interaction diameter of $16 \mathrm{~cm}$. All these values agree with the ones proposed as reference design for the preliminary studies of the XADS

The neutron source has been calculated with a Monte Carlo code, MCNPX, with suitable models to simulate the medium energy physics spallation phenomena Results are shown in Fig. 3. The neutron energy spectrum of the source shows a peak between 1 and $2 \mathrm{MeV}$, as reported in previous works. This is due to the fact that almost $70 \%$ of the generated neutrons appear during the evaporation phase, whose physics is very similar to fission. A neutron tail above $20 \mathrm{MeV}$ is also seen, reaching values of the order of the beam proton energy. This tail accounts for $10 \%$ of the whole neutron production. Integration over the full spectrum gives a neutron yield of 11.1 neutrons per incident proton.

The neutron yield is an important parameter in order to establish a link between the spallation source and the subcritical core. Actually, this value gives the proportionality between the accelerator intensity and the rate of neutrons injected into the subcritical core. For a given value of the core reactivity, a neutron flux level will be attained in the reactor, as stated in Eq. (1).

\subsection{Neutronic analysis of the subcritical reactor}

In order to allow the on-line monitoring of the XADS reactivity level, it was recommended the use of an accelerator beam intensity with square-shaped modulation, equivalent to the sum of a constant source plus a periodic negative pulse. A beam pulse interruption of about $400 \mu \mathrm{s}$ seems suitable for the type of fuel formerly specified, because it would easily allow Prompt Neutron Decay measurements of subcriticality levels larger (in module) than 2 S (a condition largely satisfied in projected ADS, as the XADS). A $\$$ is the conventional unit for reactivity, and it corresponds to a value equal to the fraction of delayed 
neutrons in a fission. For ${ }^{239} \mathrm{Pu}$ fueled reactors, it is about 0.0023 , which is also expressed as $230 \mathrm{pcm}$.

Interruptions shorter than $400 \mu$ s would also be possible for strongly subcritical reactors, but the former value is a convenient one for a conservative estimate of the thermomechanical effects. A frequency of $1 \mathrm{~Hz}$ seems suitable for this monitoring

The neutron flux evolutions in the fuel reactor region have been simulated with MCNP for the time-dependent cases corresponding to the proton beam intensity variations, as described in the previous section. Cross-section data have been taken from ENDFB-VI Results are shown in Fig. 4. It can be seen that the evolution calculated with detailed stochastic computation (Monte Carlo) is very similar to the analytical results from the diffusion equation (deterministic approach).

Fig. 4 shows the neutron flux evolution along time after the initial transient (lasting about $30 \mathrm{~s}$ in this case) has finished. It is clearly seen that the flux almost vanishes when the beam intensity is set to zero, and it goes up to the steady-state value when the beam becomes again the nominal one. It is very important to take into account that this behavior is largely dominated by the prompt neutrons, because the subcriticality margin is very high. In fact the effective multiplication factor in our case is $K_{\text {eff }}=$ 0.97 . As the delayed-neutron fraction is $\beta=334 \mathrm{pcm}$, the reactivity is $-9.57 \mathrm{~S}$.

Monte Carlo numerical simulations are indeed very accurate (if properly defined) but they do not give a clear physical insight of the physics of the problem. In order to have a good complementary analytical explanation, the diffusion equation has been solved for the slab reactor under consideration, using an integrated approach in neutron energy (one-group theory) and assuming that all fission neutrons appear as prompt ones.
The diffusion equation for the time-dependent flat plate case is

$$
\begin{gathered}
\frac{1}{v} \frac{\partial \Phi(x, t)}{\partial t}=v \Sigma_{\mathrm{f}} \Phi(x, t)-\sum_{\mathrm{a}} \Phi(x, t)+D \frac{\partial^{2} \Phi(x, t)}{\partial x^{2}} \\
+S \delta(x)
\end{gathered}
$$

As already said, it has been considered the delayed neutrons to be born as prompt ones, avoiding the resolution of the coupled set of equation generally solved in detailed kinetic analysis. In actual reactors, the effect of delayed neutrons implies a smoothing of the neutronic response to transients by the enlargement of the effective neutron lifetime in the nuclear system. Neglecting such contributions will lead to slightly sharper neutron population change, and slightly higher slopes in thermal production transients and its corresponding thermo-mechanical stresses. Therefore, our assumption is conservative, particularly from the point of view of thermo-mechanical analysis. Although delayed neutrons should be taken into account in detailed kinetic studies devoted to safety analysis of a given ADS, they have a very small contribution to the thermal response of the system.

The general solution of the diffusion equation can be expressed in a simplified way using the following expressions, which correspond to a bare fast reactor with an effective thickness yielding the same $K_{\text {eff }}$ as the reactor (with reflector) under consideration. In our case, the effective thickness, taking into account the so-called reflector saving is $206.4 \mathrm{~cm}$. With this approximation, the flux inside the fuel can be expressed in two sets of solutions, one for each time domain, i.e., the active part of the beam $(T)$, and the beam interruption $\left(t_{\mathrm{a}}\right)$ with subindex 1 and 2 , respectively. Note that $S_{n a}$ is the $n$-term of the

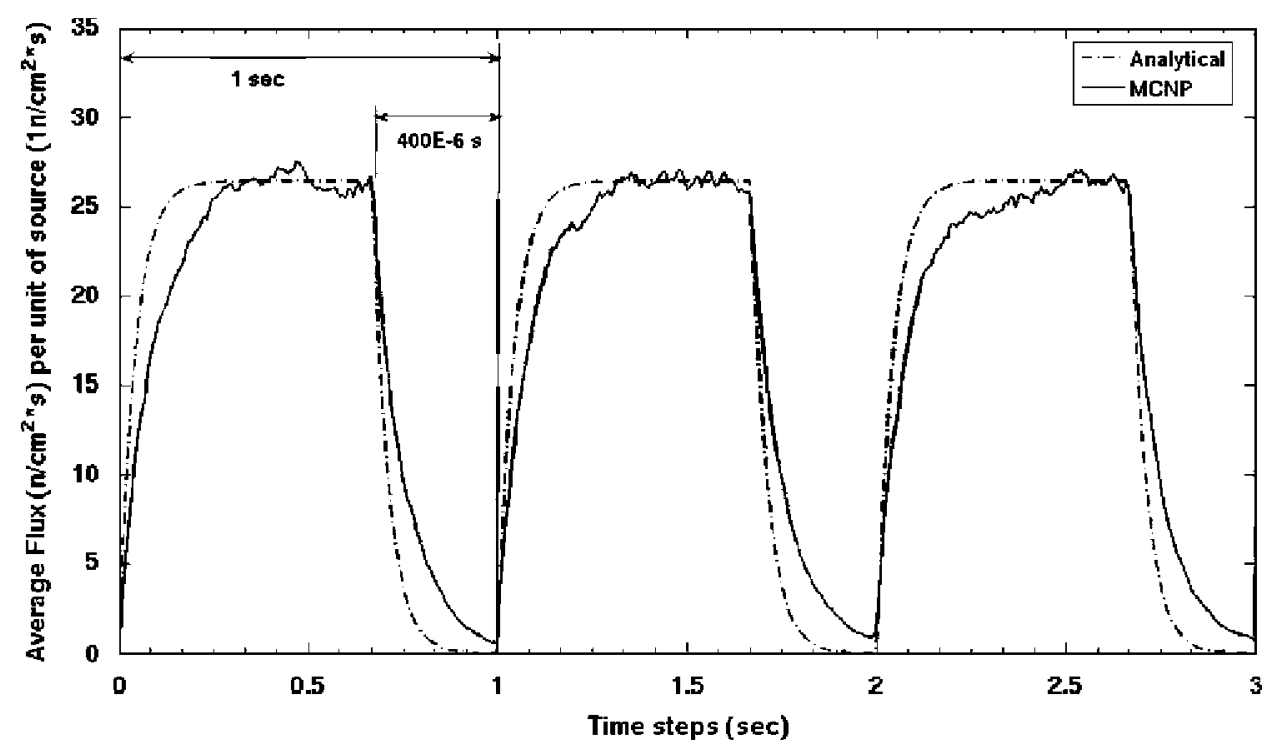

Fig. 4. Analytical and Monte Carlo results for the neutron flux in the subcritical core for $400 \mu$ s beam trips with repetition frequencies of $1 \mathrm{~Hz}$. Time variable not to scale. The neutron flux is given per source neutron emitted in the mid-plane. 
source expansion in the eigenmode series.

$$
\begin{gathered}
\Phi_{1}(x, t)=\sum A_{n_{1}}(t) \cos \left(B_{n} x\right) \\
\Phi_{2}(x, t)=\sum A_{n_{2}}(t) \cos \left(B_{n} x\right) \\
\quad t \in\left(j T, j T+t_{\mathrm{a}}\right) \\
\text { for } \quad t \in\left(j T+t_{\mathrm{a}},(j+1) T\right)
\end{gathered}
$$

where

$$
\begin{aligned}
A_{n_{1}}(t)= & A_{n_{2}}(j T) \mathrm{e}^{\left(\left(k_{n}-1\right) / l_{n}\right)(t-j T)} \\
& +\frac{v S_{n \mathrm{a}} l_{n}}{\left(1-k_{n}\right)}\left(1-\mathrm{e}^{\left(\left(k_{n}-1\right) / l_{n}\right)(t-j T)}\right) \\
A_{n_{2}}(t)= & A_{n_{1}}\left(j T+t_{\mathrm{a}}\right) \mathrm{e}^{\left(\left(k_{n}-1\right) / l_{n}\right)\left(t-j T-t_{\mathrm{a}}\right)} \\
& +\frac{v S_{n \mathrm{a}} l_{n}}{\left(1-k_{n}\right)}\left(1-\mathrm{e}^{\left(\left(k_{n}-1\right) / l_{n}\right)(t-j T)}\right) .
\end{aligned}
$$

For each eigenmode $(n)$, former parameters are defined in terms of the effective thickness, $H$, the characteristic diffusion length, $L c^{2}$, and the following neutronic parameters:

- mean number of produced neutrons per fission;

- fission macroscopic cross-section;

- absorption macroscopic cross-section.

The following expressions are obtained:

$$
\begin{aligned}
& B_{n}=\frac{(2 n+1) \pi}{H} \\
& K_{n}=\frac{v \sum_{\mathrm{f}}}{\sum_{\mathrm{a}}} \frac{1}{1+L c^{2} B_{n}^{2}} \\
& l_{n}=\frac{1}{v \Sigma_{\mathrm{a}}\left(1+L c^{2} B_{n}^{2}\right)} .
\end{aligned}
$$

Constants $A_{n 1}$ and $A_{n 2}$ are the amplitudes of the $n$ eigenfunction for each time domain and can be defined recursively from initial conditions. It is very important to note that both amplitudes, as the neutron lifetimes and the effective multiplication factors of the $n$-eigenfunction, strongly decrease as the mode-order increases. Figs. 5-7

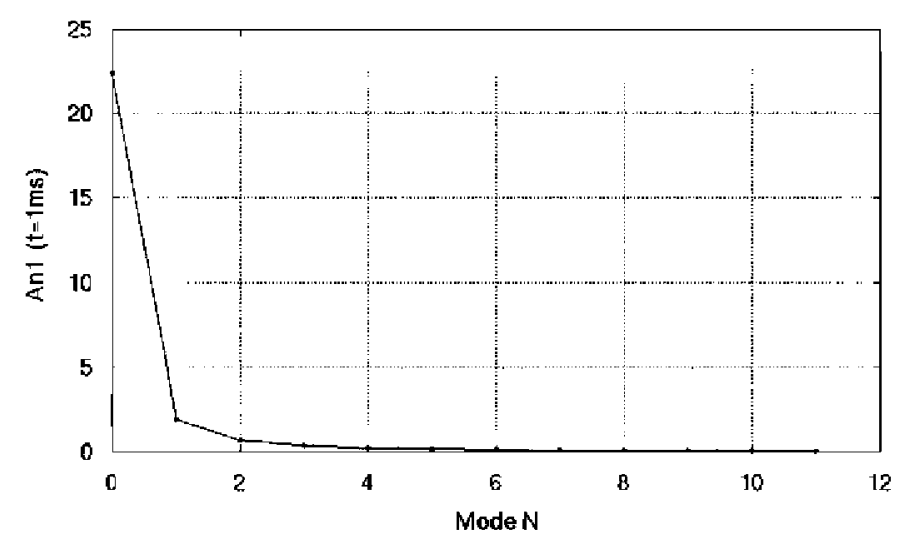

Fig. 5. Reduction of the eigenfunction amplitude $A_{\mathrm{n} 1}$ with the mode order at $1 \mathrm{~ms}$.

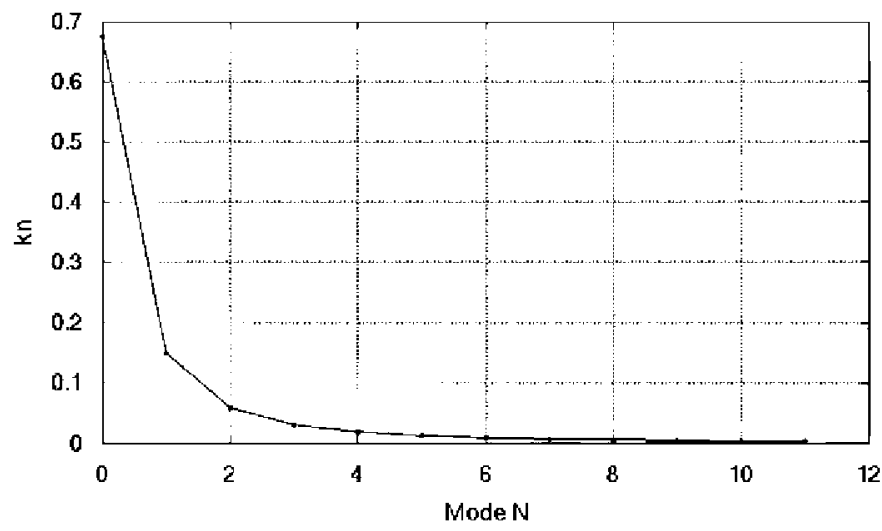

Fig. 6. Reduction of the effective multiplication factor with the mode order at $1 \mathrm{~ms}$.

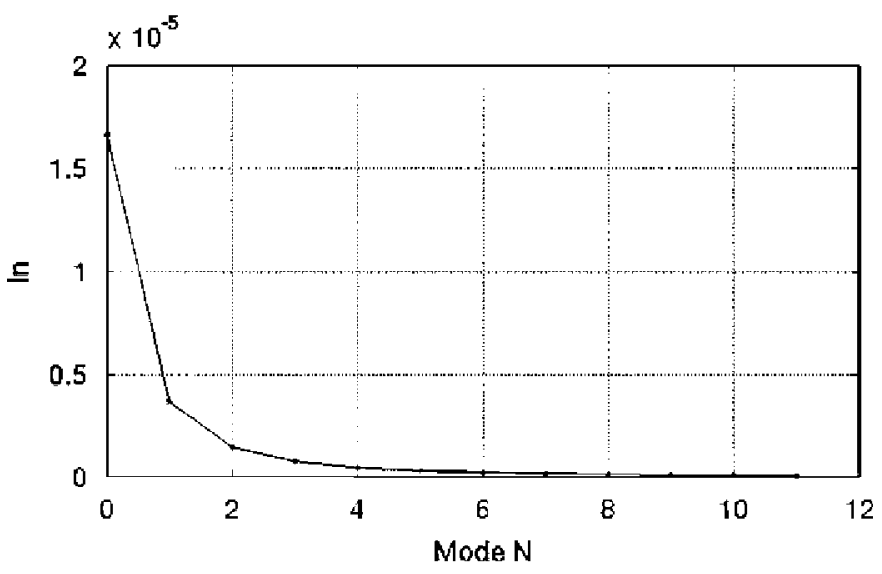

Fig. 7. Reduction of the neutron lifetime with the mode order.

show this trend. They imply that the former series actually have a very few relevant terms.

It is seen in Fig. 4 that the flux evolution mainly depends on the beam intensity, going down from the nominal value, corresponding to $100 \%$ intensity, to almost zero in the beam interruptions. In Monte Carlo simulations it is not zero because of the delayed neutrons. Even so, the flux decreases by a factor much larger than 10 along the interruption, so making it possible to measure the absolute reactivity of the reactor. In the analytical solution the flux actually vanishes because of the assumption of considering all the fission neutrons as prompt ones. Both solutions are pretty much the same, although the numerical simulation presents softer profiles along the transients.

On the other hand, it must be pointed out that the thermal power in the core will be proportional to the neutron flux in the fuel material, which will have an evolution very similar to the one depicted in Fig. 4. As a matter of fact, there will be some delay in the variations that will be longer for the fuel pins located far from the source (far from the middle plane, in our case).

In our reactor model, it has been considered that there was a mixture of fuel and coolant as reactor material. Actually, both materials will not be mixed, and the fuel will 
always be inside the cladding. However, neutron meanfree-paths will be much larger than the size of the fuel fin diameter and the fuel pin pitch, and both materials could be considered as homogenized for neutronic calculations. Therefore, the thermal power density $\left(\mathrm{W} / \mathrm{m}^{3}\right)$ evolution that will be used in the thermo-mechanical analysis will be proportional to the neutron flux evolution given by the Monte Carlo simulation. Of course it will have to be properly normalized to the nominal power density in the corresponding fuel pin. For safety reasons, the selected fuel pin has to be the one with the maximum power, in the closest place to the source.

\section{Thermo-mechanical reaction of the fuel pin cladding}

Along any thermal transient in the system, the size of the different parts will have to adapt to the new values of temperatures by means of contractions and expansions. Those mechanisms will induce cyclical strains and stresses because of the periodical nature of the monitoring pulse. These effects must be calculated to guarantee that, for the predicted conditions of the pulse, the structure will keep its mechanical integrity.

The first step in this quest is to determine the evolution of the heat flux and the field of temperatures in the reference pin, assuming the coolant is working at nominal conditions and temperature. The second step will be to determine the induced thermo-mechanical stresses, and to assess that they are not high enough to produce the breakage of the containment elements, or to cause fatigue failure.

\subsection{Thermal analysis}

It will be assumed that the thermal-nuclear feedback is negligible during the transient, and the neutron crosssections do not change along it. This assumption will be verified afterwards, because it will be found that oscillations in temperature during the transients are extremely low. This fact allows us to solve the thermal problem without recalculating the neutronics of the reactor. As already said, the evolution of the fuel power density will be the same as the flux evolution during the transient, as already calculated by Monte Carlo simulation.

In Table 2, two sets of thermal properties are shown, one for $\mathrm{Zr}$, which can be used as cladding and as a matrix for embedding actinides to be transmuted, and another for

Table 2

Main thermal properties of selected fuel matrixes (units in c.g.s. system)

\begin{tabular}{lcl}
\hline Property & $\mathrm{UO}_{2}$ & $\mathrm{Zr}$ \\
\hline Density $\left(\mathrm{g} / \mathrm{cm}^{3}\right)$ & 10.5 & 6.5 \\
Thermal conductivity $(\mathrm{W} / \mathrm{cm} \mathrm{K})$ & 0.042 & 0.227 \\
Specific heat $(\mathrm{J} / \mathrm{g} \mathrm{K})$ & 0.28 & 0.278 \\
Thermal diffusivity $\left(\mathrm{cm}^{2} / \mathrm{s}\right)$ & 0.015 & 0.125 \\
\hline
\end{tabular}

$\mathrm{UO}_{2}$, a fuel widely used in commercial reactors nowadays. Of course, future ADS fuels will be made of different materials, still under research, but these sets account for a metallic base and a ceramic one.

A thermal transient is characterized by a Fourier number of about 0.16 , the Fourier number $\left(F o\right.$ ) being $4 \alpha \tau / L^{2}$, where $\tau$ stands for time, $L$ is the characteristic length of the material and $\alpha$ is its thermal diffusivity. In a ceramic fuel pin, $L \sim 0.2 \mathrm{~cm}$ and $\alpha=0.01 \mathrm{~cm}^{2} / \mathrm{s}$, the transient time will about $0.64 \mathrm{~s}$, much longer than the neutron transient duration. In the case of having a metallic matrix, diffusivity values will be much larger and the corresponding transient times much shorter. Nevertheless, they will be orders of magnitude longer than the characteristic neutron lifetime. If it is considered at fuel pin level (with a radius of a few millimeters) the transient time for a $\mathrm{Zr}$ matrix will be about $0.02 \mathrm{~s}$.

The former values give a clear indication of the importance of the thermal effects produced by long interruptions of the accelerator beam (if the accelerator suffers from lack of reliability). If the interruption is, at least, one order of magnitude shorter than the thermal transient time, the temperature variations will be very localized and small. Let $S_{\mathrm{p}}$ stand for the specific power variation in the fuel (due to variations in the neutron flux level, which will be produced by variations in the beam current), $C_{p}$ for the fuel specific heat, and $\tau_{\mathrm{t}}$ for the transient time caused by the beam interruption. The sudden change in temperature can be roughly estimated by

$\Delta T=\frac{S_{\mathrm{p}} \tau_{\mathrm{t}}}{C_{p}}$

The exact thermal analysis requires to solve the heat transfer differential equation:

$\rho C_{p} \frac{\mathrm{d} T}{\mathrm{~d} t}=\nabla(K \nabla T)+Q$

where $Q$ is the volumetric heat density generated by nuclear reactions, and $K, \rho, C_{p}$ are the thermal conductivity, density and specific heat of the material.

In our case, the pin thermal analysis has been carried out solving the one-dimensional heat transfer equation in the radial coordinate. Eliminating the axial dependence is a standard approach due to the enormous length/diameter ratio. Moreover, as a consequence of the symmetry of the problem, angular dependence can also be eliminated.

Four temperatures are of particular importance in this analysis: The one in the center of fuel pellet, another in the periphery (close to the gap), and two other in the inner and outer surfaces of the cladding. The LBE coolant is assumed to be circulating at nominal speed along the cladding, with a temperature of $350{ }^{\circ} \mathrm{C}$. This value is assumed to remain constant along the transient, which will be much shorter than the total circulation time of the coolant along the circuit (Fig. 8).

Results of the numerical solution are shown in Fig. 9, which corresponds to the flux evolution of Fig. 4. It must 
Table 1

Fuel composition

\begin{tabular}{ll}
\hline Isotope & $\%$ weight \\
\hline${ }^{234} \mathrm{U}$ & $9.0214 \mathrm{E}-04$ \\
${ }^{235} \mathrm{U}$ & $2.3805 \mathrm{E}-02$ \\
${ }^{236} \mathrm{U}$ & $1.3417 \mathrm{E}-03$ \\
${ }^{238} \mathrm{U}$ & $4.4092 \mathrm{E}+00$ \\
${ }^{238} \mathrm{Pu}$ & $8.9909 \mathrm{E}-03$ \\
${ }^{239} \mathrm{Pu}$ & $7.8837 \mathrm{E}-01$ \\
${ }^{240} \mathrm{Pu}$ & $2.8377 \mathrm{E}-01$ \\
${ }^{241} \mathrm{Pu}$ & $6.8546 \mathrm{E}-02$ \\
${ }^{242} \mathrm{Pu}$ & $4.1972 \mathrm{E}-02$ \\
${ }^{241} \mathrm{Am}$ & $3.9220 \mathrm{E}-02$ \\
${ }^{16} \mathrm{O}$ & $1.1333 \mathrm{E}+01$ \\
${ }^{206} \mathrm{~Pb}$ & $9.1707 \mathrm{E}+00$ \\
${ }^{207} \mathrm{~Pb}$ & $8.4096 \mathrm{E}+00$ \\
${ }^{208} \mathrm{~Pb}$ & $1.9939 \mathrm{E}+01$ \\
${ }^{209} \mathrm{Bi}$ & $4.5481 \mathrm{E}+01$ \\
\hline
\end{tabular}
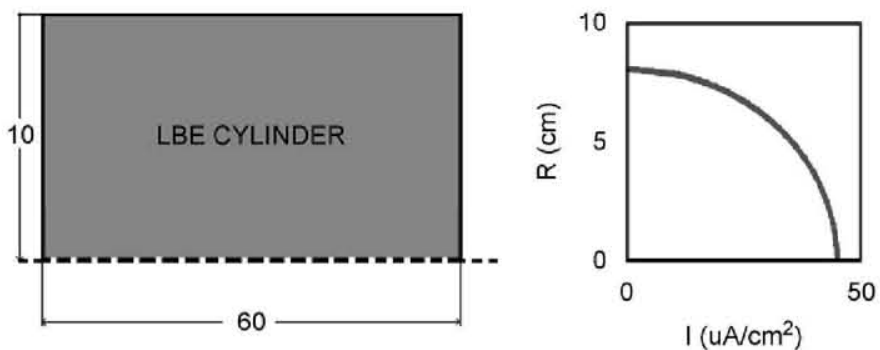

Fig. 2. The spallation source is a LBE cylinder, $60 \mathrm{~cm}$ long and $10 \mathrm{~cm}$ radius. Beam radial intensity $\left(\mu \mathrm{A} / \mathrm{cm}^{2}\right)$ profile is shown in the right-hand side.

Although a simplified source has been used in the one-dimensional reactor model, its features have been calculated using the description of spallation sources usually designed for ADS, as those of the PDS-XADS project. Main features of the source are described below.

\subsection{The neutron source}

The source has been modeled as a cylinder made of LBE (see Fig. 2) irradiated by a beam of $600 \mathrm{MeV}$ protons impinging along the axis with a cross-section distribution as the one given also in Fig. 2. This source has been used to determine the neutron generation rate and spectrum, as well as the angular distribution throughout the different surfaces. These parameters, obtained with MCNPX, have been used to define the simplified source that feeds the core. LBE was chosen as the target material for its remarkable properties as a suitable molten metal coolant with a low neutron capture. On the contrary, it has a high rate of ${ }^{210} \mathrm{Po}$ generation, which is a non-negligible problem of radiological protection (requiring coolant purification and radioactive products confinement).

For the design of the beam we have assumed a current intensity of $6 \mathrm{~mA}$, elliptically distributed, with a spot

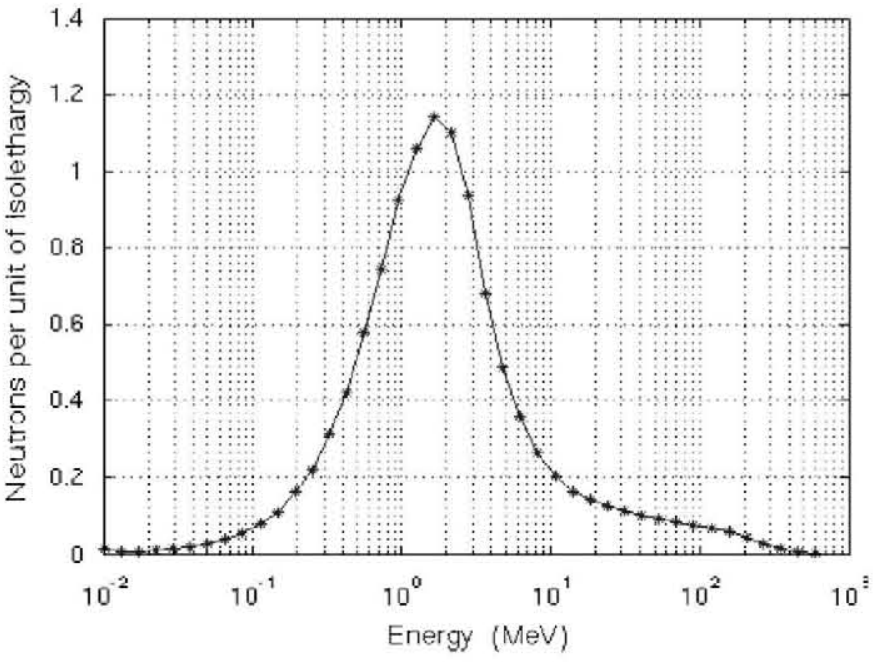

Fig. 3. Neutron spectrum of the spallation target.

interaction diameter of $16 \mathrm{~cm}$. All these values agree with the ones proposed as reference design for the preliminary studies of the XADS

The neutron source has been calculated with a Monte Carlo code, MCNPX, with suitable models to simulate the medium energy physics spallation phenomena Results are shown in Fig. 3. The neutron energy spectrum of the source shows a peak between 1 and $2 \mathrm{MeV}$, as reported in previous works. This is due to the fact that almost $70 \%$ of the generated neutrons appear during the evaporation phase, whose physics is very similar to fission. A neutron tail above $20 \mathrm{MeV}$ is also seen, reaching values of the order of the beam proton energy. This tail accounts for $10 \%$ of the whole neutron production. Integration over the full spectrum gives a neutron yield of 11.1 neutrons per incident proton.

The neutron yield is an important parameter in order to establish a link between the spallation source and the subcritical core. Actually, this value gives the proportionality between the accelerator intensity and the rate of neutrons injected into the subcritical core. For a given value of the core reactivity, a neutron flux level will be attained in the reactor, as stated in Eq. (1).

\subsection{Neutronic analysis of the subcritical reactor}

In order to allow the on-line monitoring of the XADS reactivity level, it was recommended the use of an accelerator beam intensity with square-shaped modulation, equivalent to the sum of a constant source plus a periodic negative pulse. A beam pulse interruption of about $400 \mu \mathrm{s}$ seems suitable for the type of fuel formerly specified, because it would easily allow Prompt Neutron Decay measurements of subcriticality levels larger (in module) than $2 \$$ (a condition largely satisfied in projected ADS, as the XADS). A $\$$ is the conventional unit for reactivity, and it corresponds to a value equal to the fraction of delayed 
be noted that the time scale is not constant, because the $400 \mu$ s interruption would be too short to be seen in an ordinary scale. In fact, the heating ramp would have to occupy the practical totality of the pulse time, and the cooling period would be a sudden fall. Nonetheless, the most important finding is that the temperature oscillation is totally negligible with these beam interruptions. Indeed, the oscillation is smaller than $0.5 \mathrm{~K}$ in the center of the fuel pin, and totally negligible in the outer surface (less than $0.001 \mathrm{~K}$ ).

A second interesting fact is the decoupling between the fluctuations of temperatures in the central part and the

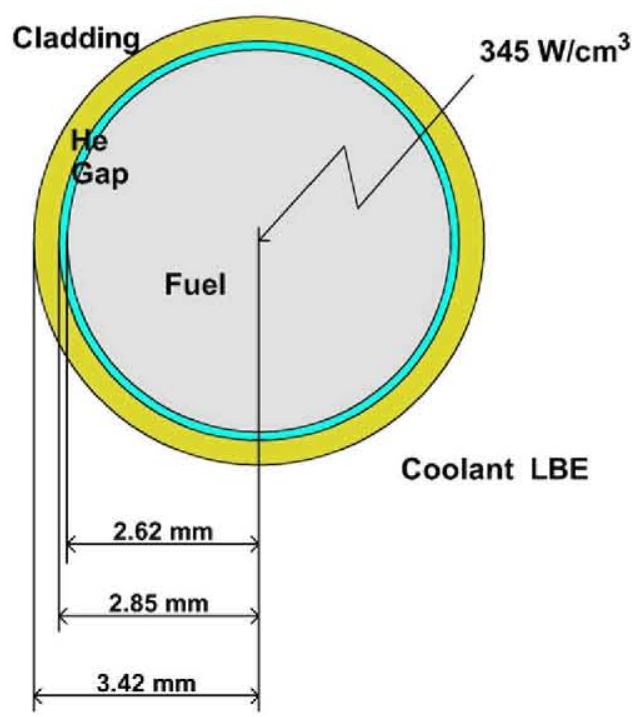

Fig. 8. Fuel pin cylindrical geometry.

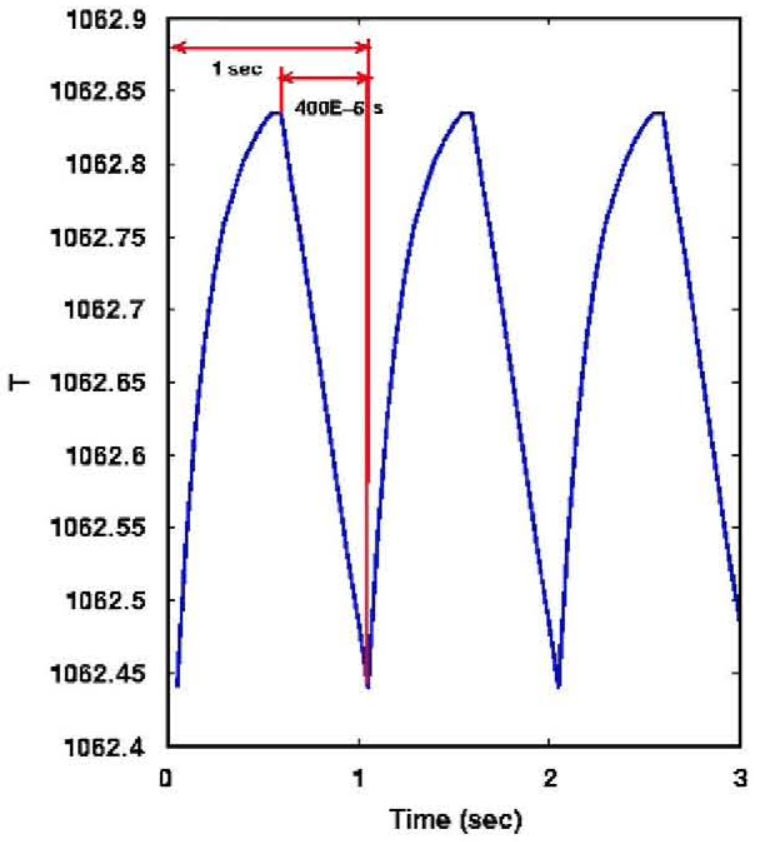

outer fuel, due to the low thermal diffusivity of uranium oxide. Although oscillations are very limited in value, they do not take place with the same phase. This delay could be the origin of possible thermal fatigue effects. Note that the central fuel node begins warming up as soon as the neutron source restores its nominal value after a beam interruption, whereas the outer part is still in the cooling phase for a short time. Similarly, inner and outer temperatures of the cladding evolve decoupled during the transient, and the temperature gradient can be much higher than the nominal one. This effect could create stresses that surpass the elastic limit or originate a fatigue phenomenon leading to the breakage of the material even with strains smaller than the elastic limit.

\subsection{Stress analysis}

The biggest problem associated to fatigue is mainly associated to the thermal gradients inside the cladding. Those affecting the fuel pellet do not suppose an immediate risk because it would be confined in the cladding, even if a pellet suffers some cracks.

It must be remembered that the expression relating stresses to temperature differences between inner and outer surfaces of the same plate or hollow cylinder is the following one:

$\frac{\sigma}{E}=\frac{\beta \Delta T}{2(1-v)}$

where $\Delta T$ is the temperature difference between both faces, $E$ is Young modulus, $v$ is Poisson module and $\beta$ is the thermal linear expansion coefficient of the material. The former equation has to embody a correction factor

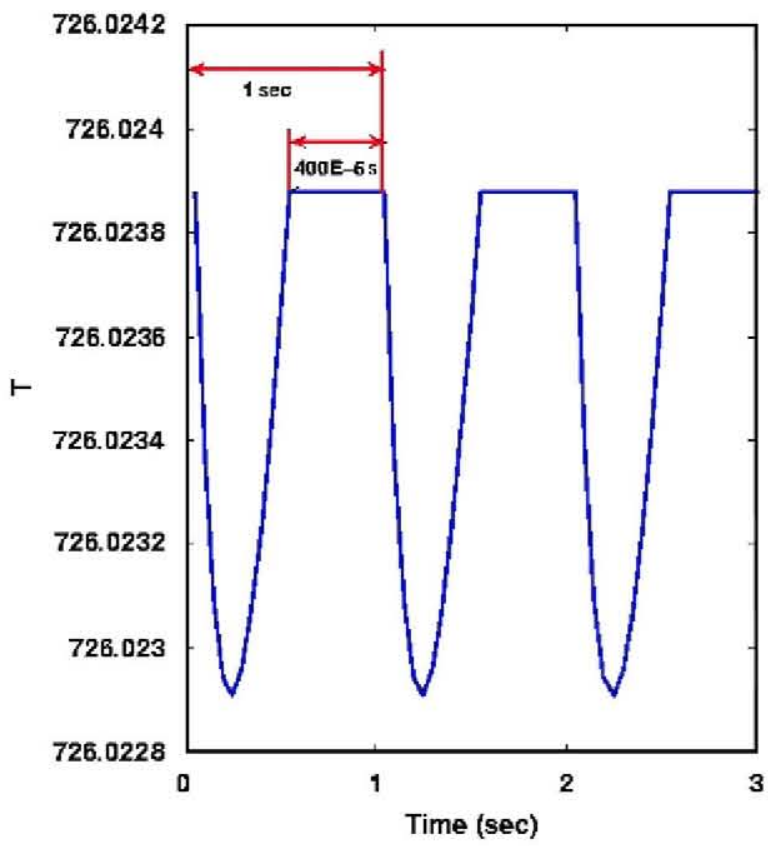

Fig. 9. Evolution of the temperatures in different nodes for the case of $1 \mathrm{~Hz}$ frequency repetition pulse. Time variable not to scale (read next paragraph for explanation). 
be noted that the time scale is not constant, because the $400 \mu$ s interruption would be too short to be seen in an ordinary scale. In fact, the heating ramp would have to occupy the practical totality of the pulse time, and the cooling period would be a sudden fall. Nonetheless, the most important finding is that the temperature oscillation is totally negligible with these beam interruptions. Indeed, the oscillation is smaller than $0.5 \mathrm{~K}$ in the center of the fuel pin, and totally negligible in the outer surface (less than $0.001 \mathrm{~K}$ ).

A second interesting fact is the decoupling between the fluctuations of temperatures in the central part and the

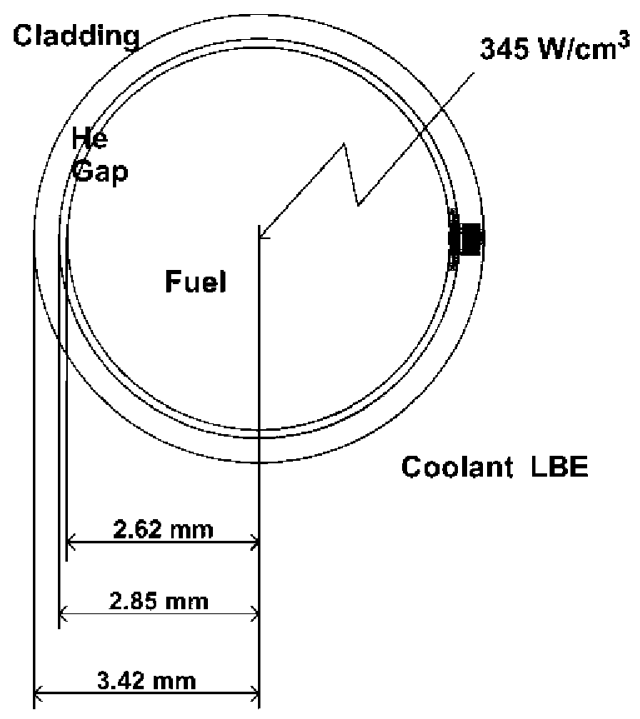

Fig. 8. Fuel pin cylindrical geometry.

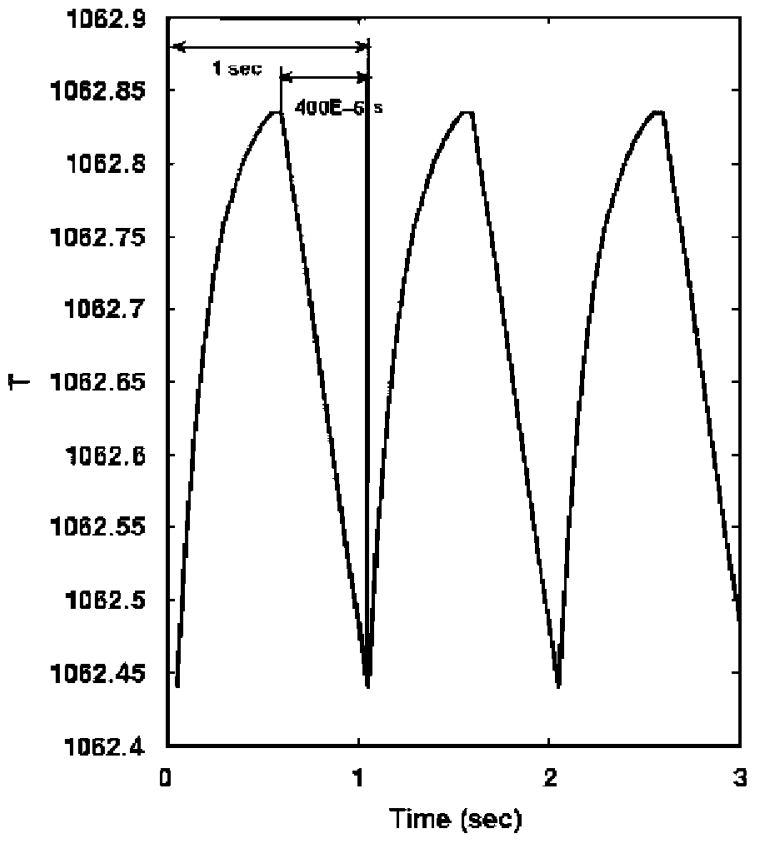

outer fuel, due to the low thermal diffusivity of uranium oxide. Although oscillations are very limited in value, they do not take place with the same phase. This delay could be the origin of possible thermal fatigue effects. Note that the central fuel node begins warming up as soon as the neutron source restores its nominal value after a beam interruption, whereas the outer part is still in the cooling phase for a short time. Similarly, inner and outer temperatures of the cladding evolve decoupled during the transient, and the temperature gradient can be much higher than the nominal one. This effect could create stresses that surpass the elastic limit or originate a fatigue phenomenon leading to the breakage of the material even with strains smaller than the elastic limit.

\subsection{Stress analysis}

The biggest problem associated to fatigue is mainly associated to the thermal gradients inside the cladding. Those affecting the fuel pellet do not suppose an immediate risk because it would be confined in the cladding, even if a pellet suffers some cracks.

It must be remembered that the expression relating stresses to temperature differences between inner and outer surfaces of the same plate or hollow cylinder is the following one:

$\frac{\sigma}{E}=\frac{\beta \Delta T}{2(1-v)}$

where $\Delta T$ is the temperature difference between both faces, $E$ is Young modulus, $v$ is Poisson module and $\beta$ is the thermal linear expansion coefficient of the material. The former equation has to embody a correction factor

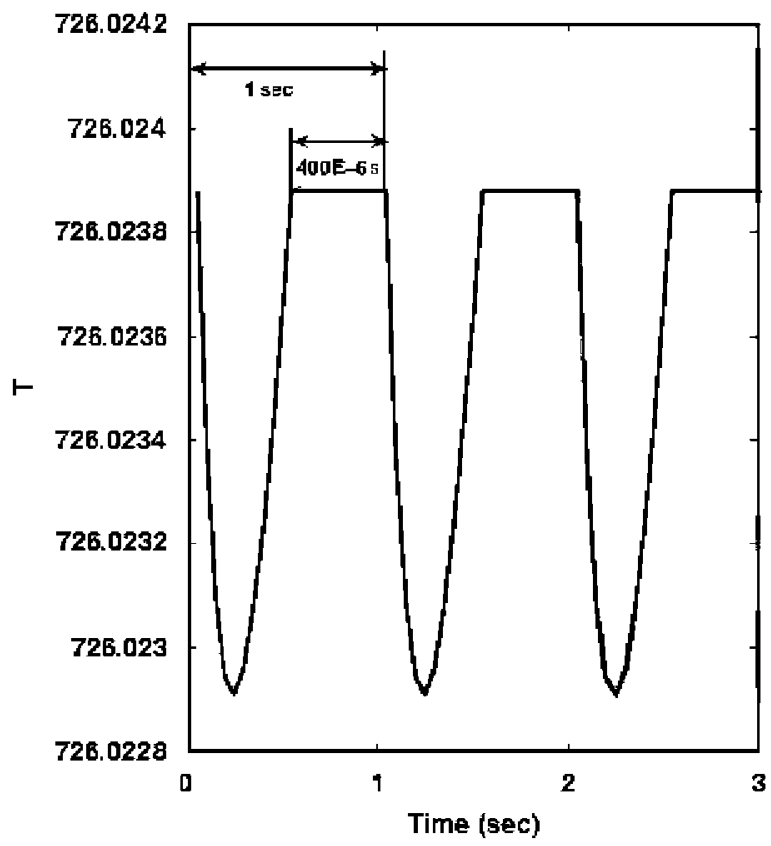

Fig. 9. Evolution of the temperatures in different nodes for the case of $1 \mathrm{~Hz}$ frequency repetition pulse. Time variable not to scale (read next paragraph for explanation). 
depending on the boundary conditions and the exact configuration of the plate or the cylinder, but it is a very good analytical estimate, and gives a good evaluation of the order of the so-called thermal stresses, which will depend on the cladding material properties.

Martensitic stainless steels have been developed for both in-core applications in advanced liquid metal fast breeder reactors (LMFBR) and for first wall and structural materials applications for commercial fusion reactors

Tungsten-stabilized martensitic stainless steels have appropriate properties for high neutron-fluence applications, including low after-heat and low radioactive inventory. HT-9 alloy has been tested for LMFBR applications and has shown very positive features in radiation damage, and creep and swelling resistance. It is a primary candidate for cladding in the current US fast reactor designs. Table 3 presents some fundamental properties of HT-9, that have been used in thermal-stress analysis.

From calculations on the fuel pin cylindrical model, it is found that the cladding hoop stress has an average value of $5 \mathrm{MPa}$. Additionally, the evolution of the hoop stress is depicted in Fig. 10, as the difference between its actual time-dependent value and the average stress, for the $1 \mathrm{~Hz}$ frequency pulse with $400 \mu \mathrm{s}$ interruptions. These oscilla-

Table 3

Thermal and structural properties of HT-9 alloy

\begin{tabular}{ll}
\hline Property & Value \\
\hline Thermal conductivity $(\mathrm{W} / \mathrm{m} \mathrm{K})$ & 28 \\
Modulus of elasticity (GPa) & 160 \\
Poisson's ratio & 0.33 \\
Coefficient of thermal expansion $\left(\mathrm{K}^{-1}\right)$ & $12.5 \mathrm{E}-6$ \\
\hline
\end{tabular}

tions are about $6.35 \mathrm{~Pa}$, which seems a rather low value. These results will be used to calculate the fatigue limit.

At first, former data could be used straightforward in the Wohler diagrams for the aforementioned material, in order to find out the number of cycles that the cladding would support before failure. However, the application of this method at high temperatures needs an elastic analysis with corrections for creep and plasticity. This method is based on article T-1432 of Appendix T of Subsection NH of the ASME Boiler and Pressure Vessel Code. For a given peak strain and a given peak temperature, this method gives the maximum allowable number of cycles that the material can afford. An important problem with this appendix is that it only includes data for the following materials: 304 stainless steel, 316 stainless steel, $\mathrm{Ni}-\mathrm{Fe}-\mathrm{Cr}$ alloy $800 \mathrm{H}$, and $21 / 4 \mathrm{Cr}-1$ Mo steel. To estimate fatigue limits for HT-9 at elevated temperatures, it was decided to use the Stromyer-type regression curve determined by the standard method , for 316 SS, as follows:

$\sigma_{\mathrm{a}}=10,775 N_{\mathrm{f}}^{-0.6194}+185.75$.

After analyzing the load pads with this equation for 316 SS properties, the allowable number of cycles must be multiplied by a factor, fHT-9, to get the allowable number of cycles for HT-9. Values for fHT-9 were evaluated for both HT-9 and 316 SS using Section 3, Subsection NB treatment, and no correction for elevated temperature operation In these cases, the allowable number of cycles for HT-9 tended to be about one sixth of the allowable number of cycles for $316 \mathrm{SS}$. Thus, the value used for fHT-9 was taken as 1/6. For higher accuracy, the effect of mean stress on fatigue life can be introduced. A variation of the Goodman model was also proposed by Wire to evaluate the equivalent stress

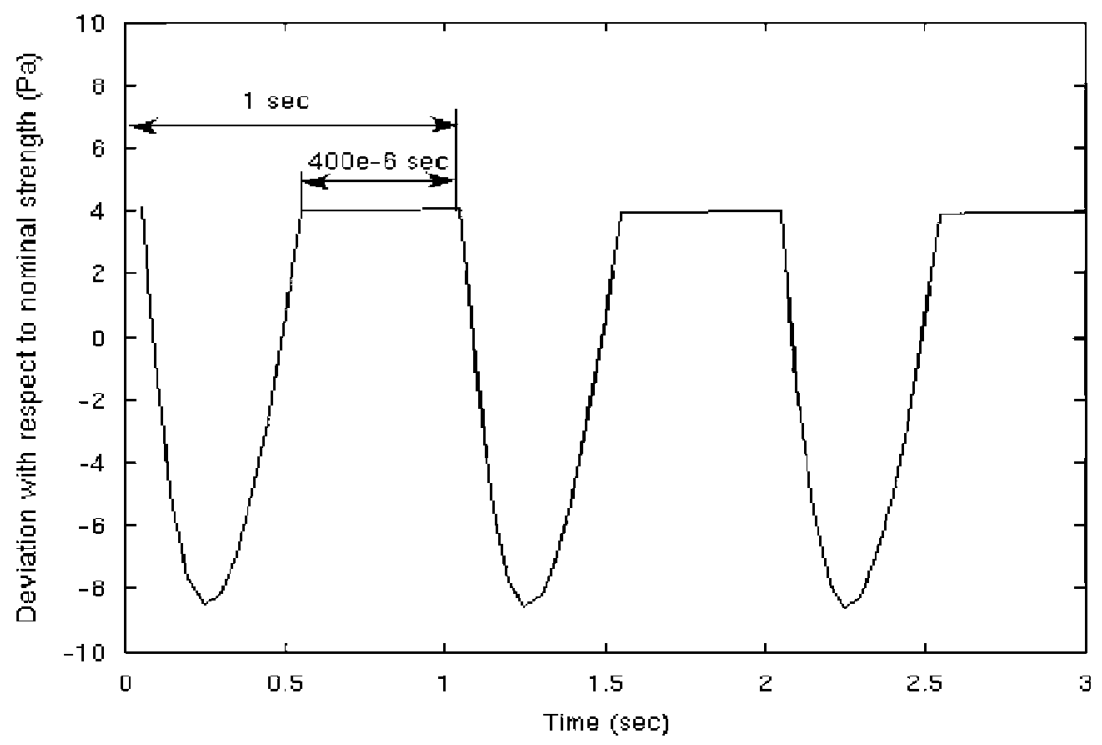

Fig. 10. Difference between the stress during the pulses in the cladding and the average stress, for the $1 \mathrm{~Hz}$ frequency pulse, with $400 \mu$ interruptions. Again time variable not to scale. 
amplitude, $\sigma_{\text {eq }}$ :

$\sigma_{\mathrm{eq}}=\frac{\sigma_{\mathrm{a}}}{1-\left(\sigma_{\mathrm{m}} / \sigma_{\mathrm{u}}\right)^{2}}$

where $\sigma_{\mathrm{m}}$ and $\sigma_{\mathrm{a}}$ are, respectively, the mean and amplitude stresses calculated previously, and $\sigma_{\mathrm{u}}$ is the ultimate strength. For HT-9 the latter takes a value of $825 \mathrm{MPa}$. With this negligible correction, the stress amplitude would not suffer any change. For all this, and since the stress amplitude is much smaller than the $187 \mathrm{MPa}$ of fatigue limit fixed by the Stromyer regression curve, it can be concluded that the cladding will preserve its integrity for a $1 \mathrm{~Hz}, 400 \mu \mathrm{s}$ interruption monitoring pulse indefinitely.

Another problem could be related to cladding loads surpassing the elastic limit at the working temperature. This problem comes from the decoupling produced in the evolution of the temperatures of the different parts of the cladding. In fatigue phenomena, failure mainly depends on thermal gradients and not on temperature. Unlike it, the elastic limit decreases as the working temperature of the cladding increases, even by a factor 2 at $300^{\circ} \mathrm{C}$. However, this effect is of very small importance in this case, since the strains that appear by the decoupling in the evolution of temperatures are absolutely negligible, about nine orders of magnitude below the $750 \mathrm{MPa}$ that is the elastic limit at cold.

\section{Conclusion}

A main safety requirement in $\mathrm{ADS}$ is to keep a specified subcriticality level in the reactor, which implies to make absolute reactivity measurements very often. This requirement can be met by making very short beam interruptions in the beam, because the flux decay could be recorded and interpreted according to any suitable method, as PJ or any other.

Those flux variations will induce similar variations in the fuel power density, which in turn will produce variations in the temperature distribution inside the fuel pin and its cladding.

For a given frequency, amplitude and width of the beam pulses, all relevant thermal features can be calculated, starting from the neutron evolution and arriving to the thermal stresses induced by temperature gradients inside a piece of material. A main risk is connected to the cyclical nature of the beam interruptions, which can activate fatigue phenomena and can produce the breakage of fuel pin claddings even for stresses smaller than the yield strength.

The full set of mechanisms (source neutron generation, neutronic reaction in the reactor, power variations, thermal stresses and strains, and mechanical assessment of those effects) have been taken into consideration to verify that beam pulsed interruptions required for reactivity monitoring would not represent an unacceptable risk for cladding integrity.
For $400 \mu$ s interruptions every second, as proposed in the EUROTRANS project, it has been verified that those cyclical thermo-mechanical stresses will not convey those risks, and their effects will remain far from reaching fatigue and elastic limits.

\section{Acknowledgments}

This work was done under the auspices of "Federico Goded Chair", UPM-CSN (Spain) and it is a part of UPM work in IP-EUROTRANS a European Union FP6 Project, FI6W-CT-2004-516520.

\section{References}

C. Rubbia, A high gain energy amplifier operated with fast neutrons, in: International Conference on Accelerator-Driven Transmutation Technologies and Applications, Las Vegas, AIP Conf. Proc. 346(1994).

C. Bowman, E. Arthur, et al. Accelerator-driven transmutation of high-level waste from the defense and commercial sectors, LA-UR96-2833, September 1996.

C.M. Van Atta, J.D. Lee, W. Heckrotte, The electronuclear conversion fertile to fissile material, Lawrence Livermore Laboratory Report, UCRL-52144, 1970.

C. Rubbia, et al., Conceptual design of a fast neutron operated high power energy amplifier, c/AT/95-44, 1995

R. Serber, Phys. Rev. 72 (1947) 1144.

D. Ridikas, W. Mittig, Nucl. Instr. and Meth. A 418 (1998) 449.

A.C. Mueller, Nucl. Phys. A 751 (2005) 453c.

J.L. Biarrotte, et al., A reference accelerator scheme for ADS applications, Nucl. Instr. and Meth. A March (2006)

P. Ravetto, M.M. Rostagno, G. Bianchini, M. Carta, A. D'Angelo, Nucl. Sci. Eng. 148 (1) (2004) 79.

A.W. Rineiski, W. Maschek, Ann. Nucl. Energy 32 (2005) 1348.

Xu Cheng, Abdalla Batta, Nan-Il Tak, Ferry Roelofs, Pablo T. León López, Nucl. Instr. and Meth. A 562 (2006) 855.

F. Sordo, P.T. Leon, J.M. Martínez-Val, Nucl. Instr. and Meth. A 574 (2007) 232.

F. Dunn, Design criteria and mitigation options for thermal fatigue effects in ATW blankets, in: Proceedings of the IAEA Technical Committee Meeting on Core Physics and Engineering Aspects of Emerging Nuclear Energy Systems for Energy Generation and Transmutation, Argonne, IL, USA, November 28-December 1, 2000. G. Rimpault, et al., Core instrumentation and reactivity control of the experimental ADS, in: Proceedings of the International Workshop on P\&T and ADS Development, October 6-8, 2003, SCK Mol, Belgium.

J.L. Muñoz-Cobo, Y. Rugama, T.E. Valentine, J.T. Mihalczo, R.B. Pérez, Ann. Nucl. Energy 28 (2001) 1519.

R.B. Perez, R.S. Boot, Excitation of neutron waves by modulated and pulsed sources, in: Proceedings of the Symposium on Pulsed Neutron Research held at Karlsruhe, 10-14 May, 1965, vol. II, IAEA, Vienna, 1965, pp. 701-728.

M. Carta, A. D'Angelo, Nucl. Sci. Eng. 133 (1999) 282.

Carl-Magnus Persson, et al., Nucl. Instr. and Meth. A 554 (2005) 374

J.M. Martinez-Val, et al., Nucl. Technol. 90 (1990) 371.

A European Roadmap for developing accelerator driven systems (ADS) for nuclear waste incineration, ISBN 88-8286-008-6, April 2001 (web: www.nea.fr).

D.B. Pelowitz (Ed.), MCNPX Users Manual(2.5.0), Los Alamos National Laboratory, April 2005.

Accelerator and Spallation Target Technologies for ADS Applications [France]OECD NEA Publication, No. 5421, 2005.

J.-L. Biarrotte, et al., Nucl. Instr. and Meth. A 562 (2006) 656. 
H.W. Bertini, Phys. Rev. 131 (1963) 1801.

E.D. Arthur, The Gnash preequilibrium statistical model code, LANL Internal Report LA-UR-88-382, 1988.

F. Atchison, Spallation and fission in heavy metal nuclei under medium energy proton bombardment, in: G.S. Bauer (Ed.), Proceedings of the Meeting on Targets for Neutron Beam Spallation Source, Julich, June 11-12, 1979, Jul-Conf-34., Kernforschungsanlage Julich GmbH, Germany, 1980, pp. 17-46.

A. Abanades, et al., Nucl. Instr. and Meth. A 478 (2002) 577

A. Gandini, M. Salvatores, I. Slessarev, Ann. Nucl. Energy 27 (2000) 1147.

C. Jammes, G. Rimpault, G. Imel, Negative Pulse Techniques for online reactivity monitoring of accelerator driven systems, European Commission contract No. FIKW-CT-2001-00179, Preliminary design studies of an Experimental Accelerator-Driven System.

Cross Section Evaluation Working Group, ENDF/B-VI Summary Documentation, Report BNL-NCS-17541 (ENDF-201), in: P.F. Rose (Eds.), National Nuclear Data Center, Brookhaven National Laboratory, Upton, NY, USA; ENDF/B-VI database, 1991. 〈http:// www.nndc.bnl.gov/nndc/endf/> .

J.R. Lamarsh, Introduction to Nuclear Reactor Theory, AddisonWesley, Reading, MA, 1972.

B. Yarlagadda, Elevated Temperature mechanical properties and corrosion characteristics evaluation of alloy HT-9, J.N.T.U. College of Engineering, Hyderabad, India, 2002 (web: http://nstg.nevada.edu/ Atw/aaalibrarythesis2.html).

R.G. Pahl, C.G. Lahm, S.L. Hayes, Performance of HT9 clad metallic fuel at high temperature, Joint American Nuclear Society
(ANS)/European Nuclear Society (ENS) International Meeting on Fifty Years of Controlled Nuclear Chain Reaction: Past, Present, and Future; 15-20 November, 1992, Chicago, IL, USA.

R.J. DiMelfi, E.E. Gruber, J.M. Kramer, Microstructural Evaluation in a Ferritic-Martensitic Stainless Steel and its Relation to Hightemperature Deformation and Rupture Model, RE-207, Argonne National Laboratory, Argonne, IL, USA.

The American Society of Mechanical Engineers, ASME Boiler \& Pressure Vessel Code, an International Code, Section III, Subsection NH, Appendix T, Article T-1432, 1998.

C.E. Stromeyer, Proc. Roy. Soc. XC (1924) 411.

JSMS, Standard evaluation method of fatigue reliability for metallic materials - standard regression methods of $S-N$ curves, 2002 JSMSSD-6-02.

N. Miura, Y. Takahashi, Int. J. Fatigue 28 (2006) 1618

J. Goodman, Mechanics Applied to Engineering, Longman Green and Co., London, 1989

G.L. Wire, T.R. Leax, J.T. Kandra, ASME Pres. Ves. Piping Div. PVP 386 (1999) 213.

T.R. Leax, ASME Pres. Ves. Piping Div. PVP 386 (1999) 229.

J.U. Knebel, H. Ait Abderrahim, L. Cinotti, L. Mansani, F. Delage, C. Fazio, M. Giot, B. Giraud, E. González, G. Granget, S. Monti, A.C. Mueller, European research programme for the transmutation of high level nuclear waste in an accelerator driven system (EUROTRANS), in: Proceedings of the Ninth International Exchange Meeting on Partitioning and Transmutation, Nimes, France, September 25-29, 2006. 